\title{
USE OF SILVER NANOPARTICLES IN TREATMENT OF SOCIALLY SIGNIFICANT DISEASES
}

M.G. Grigoriev, PhD student, L.N. Babich, student,

National Research Tomsk Polytechnic University, Tomsk

634050, Tomsk, Lenin Avenue, 30, phone. +7(913) 824-83-97

E-mail: Mishatpu@mail.ru

Provision of high efficacy and quality of drugs developed based on nanotechnology is possible only in case of compliance with the requirements for the development, study, introduction and production of new pharmaceuticals. While determining the toxicological characteristics of substances, determination of genotoxicity and cytotoxicity is the first stage, the purpose of which is to determine the ability to induce primary DNA damage, as well as the possible negative effects on cell cultures. Taking into account the need for the introduction of new antimicrobial agents it is reasonable to develop combined agents that would have better efficacy and safety indicators. The studies of nanosilver-based drugs acting against antibiotic-resistant microorganisms, cytotoxicity and genotoxicity study of nanocomposite, finely dispersed silica and silver nanoparticles produced by alkaline single cell-gel electrophoresis assay are of significant scientific and practical interest. It has been demonstrated that finely dispersed silica nanocomposite with silver nanoparticles has no genotoxic properties, and its cytotoxicity disappears at concentrations below $0.007 \%$ [ $1-3]$.

Creation of functional nanomaterials and nanodevices is the most promising using the nanoparticles obtained by the chemical solution methods. These methods allow producing nanoparticles, especially from precious metals such as gold and silver, with a profusive variety of composition, structure, shapes and sizes.

Possible applications of nanoparticles are not limited to a certain range. They are widely used for diagnostics and treatment of various diseases, including cancer. They are also used in immunochemical studies. So, they have been extensively studied in a new sect of experimental medicine, which is now called "nanomedicine". According to the literature it is known that, in particular, the silver nanoparticles may be used to produce a wide range of materials with antibacterial properties. At the same time, it has been found over the past few years that different nanoparticles when they enter the body, can lead to the development of serious diseases (nanopathologies). It is also emphasized that metal nanoparticles can enter the body in different ways, for example, through the mucous membranes of the respiratory tract and digestive system and through the skin when using cosmetics. They can penetrate through the bloodstream as an ingredient of vaccines and serums, etc. The risk of the spread of diseases of that type (nanopathologies) has been still not entirely investigated or realized. However, their importance is great today and in it is obvious that it will increase in the future. The determination of the causes of "harmful" (pathological) effect of nanoparticles and development of the ways to control the manifestations of diseases associated therewith are still going on. The question raised by the penetration of nanoparticles into the body remains an open one. Therefore, this area is now becoming the subject of the new and developing sect in the modern experimental medicine [4].

Thus it can be said without prejudice that determination of additional ways, as well as the methods of nanoparticle effect is continuously going on. This especially applies to the effect of metal nanoparticles on the living organism. It is still critically important and popular in researches and developments. The need is expressed in improvement of existing prototypes, as well as production of new generations of drugs. This will be followed by the development of the new treatment methods in the field of nanomedicine. These aspects will allow revealing the features to determine the causes of nanopathologies emergence. They will also allow determining and developing science-based and acceptable ranges of concentration and size of nanoparticles, depending on their location: in water, air or as a part of various materials, which a human contacts with. Further opportunities to study the properties of silver nanoparticles are being expanded. The development of options of their practical application reaches higher heights. Determination of the mechanisms of their biological effect on the macroorganism is being improved. The method for the production thereof largely depends on them. The latter is a criterion determining in many cases their structure, sizes, physical and chemical properties. Finally everything lies in stability, which specifies the lifetime of certain nanosized particles. In recent years, among the variety of methods for production of nanoparticles the chemical synthesis methods based on the reduction of metal ions to atoms form a large group. This can occur in solutions, and in other conditions that are favorable to the subsequent aggregation of the atoms and ions to form the necessary nanoparticles [5].

The modern arsenal of pharmacotherapy has a wide range of potent drugs. However, despite this, the results of treatment are often not very satisfactory. Among the many factors that reduce the efficacy of existing drugs, it should be noted that they have extremely unfavorable biodistribution. It may be due to the specific features and difficulties in their penetration into the nidus. The underlying causes of such a pattern may include hindered access to the target organ due to the structural features of specific tissues (presence of different histo-hematic barriers). They may also include insufficient intracellular transport due to the features 
and/or physico-chemical properties of the drug. The usual way to overcome this obstacle is to increase treatment efficacy by increasing its dose. The drawback of this method of achieving therapeutic effect is surely the increase in the non-specific toxicity of the drug used. In this regard, the specified problem is of utmost importance in cases of application of potent drugs. These include, for example, antineoplastic agents and antibiotics. Their application is always associated with various side effects. It is often a factor that vitiates all efforts $[6-8]$.

In view of the aforesaid polymeric nanoparticles are of particular interest. These are the particles from 10 to $1000 \mathrm{~nm}$. Their dimension is a criterion determining stability and required capacity in respect to a broad spectrum of drugs [9].

It should be emphasized that this is a long-term use of high doses of silver (solution concentration 30 - $50 \mathrm{mg} / \mathrm{l}$ ) over many years for therapeutic purposes. It is also observed when using silver compounds under production conditions. This may lead to the deposition of silver in the skin and the subsequent change of skin color. This is called argyria, an occupational disease of jewelers when the skin is getting tanned. It results from the photochemical reduction of silver ions in the skin thickness. However, no changes in the functional state of organs and systems have been revealed when examining such patients. No abnormal findings in the biochemical processes taking place in the body have been detected as well. Only increased resistance to most viral and bacterial infections was observed in all people with symptoms of argyria [10].

Over the last years there has been certain information in domestic and foreign scientific literature that silver is a potent immunomodulator. This action is comparable only with steroid hormones. It has been also found that silver can both stimulate and inhibit the phagocytosis depending on the dose used. It has been noted, that under the action of silver particles the amount of immunoglobulin of IgA, IgM-, IgG- classes is increased and the percentage of T-lymphocytes is increased as well [11].

Thus, the analysis of the contemporary literature is indicative of an urgent need to create fundamentally new drugs for the treatment of socially significant diseases on the basis of modern nanotechnology. Nanosilverbased ointment for the treatment of infectious dermatitis, nanoiron capsules for the treatment of anemia, nanosized silica (silics) for the treatment of poisoning, lipoflamin for the treatment of myocardial infarction are already used in clinical practice today. However, there are no effective and safe drugs for the treatment of viral diseases, including HIV, hepatitis, influenza, herpes, Alzheimer's disease, Parkinson's disease, malignant tumors, infectious diseases, which are caused by antibiotic-resistant strains of microorganisms, endocrine system disorders (diabetes, myxedema) schizophrenia, epilepsy, manic-depressive illness.

References.

1. D.S. Savchenko, Study of genotoxicity and cytotoxicity of nanocomposite of finely-dispersed silica with silver nanoparticles, Journal of New Medical Technologies. 20 (2013) 44-47 (in Russian)

2. G.M. Shub, O.G. Shapoval, S.Ye. Velmakin, L.B. Sakulina, Changing of adhesive activity of Eschrichiacoli and Pseudomonasaeruginosa under the influence of silver nanoparticles, Fundamental research. 6 (2013) 1453-1455 (in Russian)

3. O. Brandt, M. Mildner, A. E. Egger, M. Groessl, U. Rix, M. Posch, B. K. Keppler, C. Strupp, B. Mueller, G. Stingl, Nanoscalic silver possesses broad-spectrum antimicrobial activities and exhibits fewer toxicological side effects than silver sulfadiazine, Nanomedicine-UK. 8 (2012) 478-488

4. M.S. Islam, C. Larimer, A. Ojha, I. Nettleship, Antimycobacterial efficacy of silver nanoparticles as deposited on porous membrane filters, Mater. Sci. Eng. C. Mater. Biol. Appl, 33 (2013) 4575-4581

5. F. Martínez-Gutiérrez, J.M. Guajardo-Pacheco, M.E. Noriega-Trevino, E.P. Thi, N. Reiner, E. Orrantia, Y. Av-Gay, F. Ruiz, H. Bach, Antimicrobial activity, cytotoxicity and inflammatory response of novel plastics embedded with silver nanoparticles, Future Microbiol, 8 (2013) 403-411

6. Ye.M. Yegorova, Biochemical synthesis of gold and zinc nanoparticles in reverse micelles, J Phys Chem, 84 (2010) 713-720 (in Russian)

7. N.B. Demina, S.A. Skatkov, Pharmaceutical nanotechnology: development of technological disciplines in higher pharmaceutical education, Farmatsiya, 2 (2009) 46-50 (in Russian)

8. Z. Marková, K.M. Šišková, J. Filip, J. Cuda, M. Kolar, K. Safarova, I. Medrik, R. Zboril, Air Stable Magnetic Bimetallic Fe-Ag Nanoparticles for Advanced Antimicrobial Treatment and Phosphorus Removal, Future Microbiol, 47 (2013) 5285-5293

9. N. Chen, Y. Zheng, J. Yin, X. Li, C. Zheng, Inhibitory effects of silver nanoparticles against adenovirus type 3 in vitro, J. Virol. Methods, 193 (2013) 470-477

10. V. Ye. Borisenko, Influence of nanosized particles on biological objects, Meditsina: theoretical and practical reviewed medical journal in Belarus, 1 (2010) 39-43 (in Russian)

11. L.S. Sosenkova, Ye.M. Yegorova, Small silver nanoparticles for studies of biological effects, J Phys Chem, 85 (2011) 1-10 (in Russian) 